\title{
Some properties of partial fidelities
}

\author{
Alexey E. Rastegin \\ Department of Theoretical Physics, Irkutsk State University, Gagarin Bv. 20, \\ Irkutsk 664003, Russia
}

\begin{abstract}
Basic properties of Uhlmann's partial fidelities are discussed. Statistical interpretation in terms of POVM measurements is established. Multiplicativity properties are considered. The relationship between partial fidelities and partitioned trace distances is derived. As it is shown, the partial fidelities cannot decrease under unistochastic quantum operations. Thus, the partial fidelities have good properties in the sense of their use as distinguishability measures.
\end{abstract}

PACS: 03.67.-a, 03.65.Ta

\section{Introduction}

The concept of fidelity provides a very useful measure of distinguishability [1, 2, 3. It is well known that all the devices for quantum information processing are inevitably exposed to noise [4. Therefore, the pure states used by us will eventually evolve to mixed states. Thus, good distance measures between mixed quantum states are needed for comparison of real and ideal processes [5]. For example, different measures of distinguishability were utilized for study of probability simplex [6], quantum entanglement [7, approximate broadcasting [8] and quantum circuit complexity [9]. If states are pure then comparison of them is not difficult. In contrast, there exist many ways to compare two mixed quantum states.

Both the fidelity and the trace distance are extensively utilized as measures of distinguishability [4, 10. However, these two measures are not able to pose the problem of state closeness in all respects. For instance, the equality of fidelities for two pairs of density operators does not imply their unitary equivalence [11. The justified answer is provided by use of the partial fidelities [11. In the literature, other fidelity measures between quantum states have been discussed [12, 13. There are also some closely related measures such as the Bures distance [6, 7, the angle [4, 14, the Monge distance [15] and the sine distance [16.

In principle, reasons for use of one or another distance measure are especially based on main properties of the measure. These properties are usually connected with the measurements, behaviour under quantum operations and the convexity (concavity) in inputs. For convenience, they must provide useful mathematical tools for the work. In addition, the properties should have clear physical interpretation. In the present paper, we discuss the partial fidelities 
introduced by Uhlmann. In Ref. 11] Uhlmann paid key attention to the equivalence of pairs of mixed states under invertible transformations of the state space. The aim of the present work is to analyze partial fidelities in those respects that are not considered in Ref. [11. In particular, quantum-classical relations and behaviour under quantum operations are examined.

\section{Definitions and background}

In this section, we shall briefly review necessary facts from linear algebra. Then the basic definitions will be given. Let $\mathcal{H}$ be $d$-dimensional Hilbert space. By $\operatorname{supp}(\mathrm{X})$ we denote the support of operator $\mathrm{X}$. Putting an inner product function for operators on $\mathcal{H}$, we define

$$
\langle\mathrm{X}, \mathrm{Y}\rangle_{\mathrm{hs}}:=\operatorname{tr}\left(\mathrm{X}^{\dagger} \mathrm{Y}\right) .
$$

This is the Hilbert-Schmidt inner product of operators $X$ and $Y$ [17. For any operator $\mathbf{X}$ on $\mathcal{H}$ the operator $\mathbf{X}^{\dagger} \mathbf{X}$ is positive, that is $\left\langle\psi\left|\mathbf{X}^{\dagger} \mathbf{X}\right| \psi\right\rangle \geq 0$ for all $|\psi\rangle \in \mathcal{H}$. The operator $|\mathbf{X}|$ is defined as unique positive square root of $\mathbf{X}^{\dagger} \mathbf{X}$. The eigenvalues of operator $|X|$ counted with their multiplicities are named singular values of operator $\mathrm{X}[17,18$. As a rule, we will deal with eigenvalues of Hermitian operator. So these eigenvalues are real numbers. In the text, the eigenvalues of operator $\mathbf{X}$ are assumed to be indexed in decreasing order, that is

$$
\operatorname{spec}(\mathrm{X})=\left\{\lambda_{1}(\mathrm{X}) \geq \lambda_{2}(\mathrm{X}) \geq \cdots \geq \lambda_{d}(\mathrm{X})\right\} .
$$

The singular values of $\mathbf{X}$ are then denoted by $s_{1}(\mathrm{X}) \geq \cdots \geq s_{d}(\mathrm{X})$. So the $k$-fidelity between two mixed states is defined as follows 11 .

Definition 1. The $k$-th partial fidelity between density operators $\rho$ and $\omega$ is the sum of $(d-k)$ smallest singular values of $\sqrt{\rho} \sqrt{\omega}$, that is

$$
F_{k}(\rho, \omega):=\sum_{j>k} s_{j}(\sqrt{\rho} \sqrt{\omega}) .
$$

Uhlmann showed [11 that the partial fidelities are symmetric, unitarily invariant and jointly concave. The proof of joint concavity is based on the following result. We consider all the pairs $\{X, Y\}$ of positive operators that satisfy

$$
\begin{aligned}
& \mathrm{XYX}=\mathrm{X}, \\
& \mathrm{YXY}=\mathrm{Y} .
\end{aligned}
$$

It have been shown that $\operatorname{rank}(X)=\operatorname{rank}(\mathrm{Y})=\operatorname{rank}(\mathrm{XY})$ [11. Then the $k$-th partial fidelity can be expressed as 11

$$
F_{k}(\rho, \omega)=\frac{1}{2} \inf \{\operatorname{tr}(\rho \mathbf{X})+\operatorname{tr}(\omega \mathrm{Y})\},
$$

where the infimum is taken over all the above pairs of rank $(d-k)$. In general, the sign "inf" cannot be replaced by the sign "min" in Eq. (6). In the case $k=0$, Definition 1 is reduced to the well-known formula for usual fidelity. Indeed, we obtain

$$
F_{0}(\rho, \omega)=\sum_{j=1}^{d} s_{j}(\sqrt{\rho} \sqrt{\omega}) \equiv \operatorname{tr}|\sqrt{\rho} \sqrt{\omega}|,
$$


and the latter is the fidelity [4, 19. The square of this value was introduced by Uhlmann as "transition probability" between two states 1. Note that Jozsa [3] used the word "fidelity" just for Uhlmann's transition probability. This is more convenient in some tasks of quantum information processing, for instance in quantum cloning [14, 20]. However, in the present paper we shall use the term "fidelity" for the expression in Eq. (7). In the physical literature the basic properties of fidelity are usually given by staying in finite dimensions. But they hold in much more generality [21].

Distinguishability measures between quantum states are closely related to the classical distinguishability measures. Let $\left\{p_{i}\right\}$ and $\left\{q_{i}\right\}$ be two probability distributions over the same index set. The fidelity between these distributions is defined by [10, 19

$$
\mathcal{F}\left(p_{i}, q_{i}\right):=\sum_{i} \sqrt{p_{i} q_{i}} .
$$

There is a direct analogy between the right-hand side of Eq. (77) and the righthand side of Eq.(8). Let integer $r$ denote the cardinality of the sets $\left\{p_{i}\right\}$ and $\left\{q_{i}\right\}$. In the same manner, the classical $k$-fidelity can be introduced as the sum of $(r-k)$ smallest numbers $\sqrt{p_{i} q_{i}}$.

Definition 2. The $k$-th partial fidelity between probability distributions $\left\{p_{i}\right\}$ and $\left\{q_{i}\right\}$ is defined by

$$
\mathcal{F}_{k}\left(p_{i}, q_{i}\right):=\sum_{i>k} \sqrt{p_{i} q_{i}} \downarrow
$$

where values $\sqrt{p_{i} q_{i}} \downarrow$ are indexed in the decreasing order.

Like the standard fidelity, the quantum $k$-fidelity are closely related to the classical $k$-fidelity for commuting density operators $\rho$ and $\omega$. In this simple case the two density operators are diagonal in the same orthonormal basis $\{|i\rangle\}$, namely

$$
\begin{aligned}
& \rho=\sum_{i} \mu_{i}|i\rangle\langle i|, \\
& \omega=\sum_{i} \nu_{i}|i\rangle\langle i| .
\end{aligned}
$$

The operator $\sqrt{\rho} \sqrt{\omega}=\sum_{i} \sqrt{\mu_{i} \nu_{i}}|i\rangle\langle i|$ has singular values $\sqrt{\mu_{i} \nu_{i}} \downarrow$. Due to Definitions 1 and 2, we at once obtain

$$
F_{k}(\rho, \omega)=\mathcal{F}_{k}\left(\mu_{i}, \nu_{i}\right)
$$

In the next section, a connection between quantum and classical will be given in terms of quantum measurements.

Now we write down some simple properties of $k$-fidelity. These facts are based on the following easy reason. Let $\left\{a_{i}\right\}$ be a set of $r$ positive numbers arranged in the decreasing order. We then define two quantity

$$
\begin{aligned}
A_{k} & :=\sum_{1 \leq i \leq k} a_{i}, \\
B_{k} & :=\sum_{k+1 \leq i \leq r} a_{i} .
\end{aligned}
$$

When the index $i$ ranges the empty set, any sum is assumed to be zero.

Lemma 3. For all $k=0,1, \ldots, r$, there holds

$$
\begin{gathered}
(k+1) A_{k} \geq k A_{k+1}, \\
(r-k-1) B_{k} \geq(r-k) B_{k+1} .
\end{gathered}
$$


Iterating Eq. (16), we see that both the classical and quantum partial fidelities obey

$$
\mathcal{F}_{k}\left(p_{i}, q_{i}\right) \leq \frac{r-k}{r} \mathcal{F}_{0}\left(p_{i}, q_{i}\right), \quad F_{k}(\rho, \omega) \leq \frac{d-k}{d} F_{0}(\rho, \omega) .
$$

Since 0-fidelity does not exceed one, we have $\mathcal{F}_{k}\left(p_{i}, q_{i}\right) \leq(r-k) / r$ and $F_{k}(\rho, \omega) \leq$ $(d-k) / d$. The last two inequalities are saturated if and only if $\left\{p_{i}\right\}=\left\{q_{i}\right\}$ and $\rho=\omega$. Further, $\mathcal{F}_{k}\left(p_{i}, q_{i}\right)=0$ for all $k$, if and only if the distributions do not overlap at all. Similarly, $F_{k}(\rho, \omega)=0$ for all $k$, if and only if the subspaces $\operatorname{supp}(\rho)$ and $\operatorname{supp}(\omega)$ do not intersect.

\section{Quantum-classical relations via measurement}

In this section, we provide a statistical interpretation for partial fidelities between density operators. Reasons are formulated in terms of probabilities generated by a quantum measurement. A general form of quantum measurement is described by so-called "positive operator-valued measure" (POVM). Recall that POVM $\left\{\mathrm{M}_{m}\right\}$ is a set of positive operators $\mathrm{M}_{m}$ satisfying the completeness relation

$$
\sum_{m} \mathrm{M}_{m}=\mathbf{1}
$$

where $\mathbf{1}$ is the identity operator on the space $\mathcal{H}[22$. For two density operators, the traces $\operatorname{tr}\left(\mathrm{M}_{m} \rho\right) \equiv p_{m}$ and $\operatorname{tr}\left(\mathrm{M}_{m} \omega\right) \equiv q_{m}$ are the probabilities of obtaining a measurement result labeled by $m$. Measuring concrete observable, we are concerned with projective measurement described by so-called "projection valued measure" (PVM) 22].

The explicit statistical interpretation for the fidelity was derived in Ref. [19]. The authors of Ref. [19] showed that the fidelity satisfies

$$
F(\rho, \omega)=\min \mathcal{F}\left(p_{m}, q_{m}\right),
$$

where the minimization is over all POVMs. So, the quantum fidelity is achievable lower bound for the classical fidelity generated by measurement. By relevant modification, this property can be extended to partial fidelities. Before the assertion, we recall a useful fact from matrix theory. In the seminal paper [23] Ky Fan obtained a lot of connected results with respect to extremal properties of eigenvalues. One of his formulations is now known as Ky Fan's maximum principle [18. This principle is one of important results in majorization theory [24. In our notation, the second part of Theorem 1 of Ref. [23] says that for any Hermitian operator $\mathrm{X}$

$$
\sum_{j>k} \lambda_{j}(\mathrm{X})=\min \{\operatorname{tr}(\Pi \mathbf{X}): \operatorname{rank}(\Pi)=d-k\},
$$

where the minimum is taken over all projectors $\Pi$ of rank $(d-k)$. For positive $\mathrm{X}$, the condition can be replaced by $\operatorname{rank}(\Pi) \geq d-k$. (Recall that we assume the decreasing order of eigenvalues.) The minimum is obviously reached by the projector $\Pi_{\min }$ onto the subspace corresponding to $(d-k)$ smallest eigenvalues of operator X. Modifying the reasons given in Ref. [23], we can state that for positive $X$,

$$
\sum_{j>k} \lambda_{j}(\mathrm{X})=\min \{\operatorname{tr}(\mathrm{TX}): \mathbf{0} \leq \mathrm{T} \leq \mathbf{1}, \operatorname{tr}(\mathrm{T}) \geq d-k\}
$$


where the minimization is over those positive operators $T$ that obey $\mathrm{T} \leq \mathbf{1}$ and $\operatorname{tr}(\mathrm{T}) \geq d-k$. We do not enter into details here.

Theorem 4. If all the POVM elements satisfy $\operatorname{tr}\left(\mathrm{M}_{m}\right) \geq 1$, then for arbitrary two density operators $\rho$ and $\omega$ there holds

$$
F_{k}(\rho, \omega) \leq \mathcal{F}_{k}\left(p_{m}, q_{m}\right) .
$$

Proof. By the polar decomposition [4, there is $|\sqrt{\rho} \sqrt{\omega}|=\sqrt{\rho} \sqrt{\omega} \bigvee$ for some unitary $\mathrm{V}$. It is known that operators $X Y$ and $Y X$ have the same eigenvalues (see, for instance, Exercise A6.5 in Ref. 4]). Therefore, we have

$$
\operatorname{spec}(|\sqrt{\rho} \sqrt{\omega}|)=\operatorname{spec}(\sqrt{\omega} \vee \sqrt{\rho}) .
$$

For given POVM $\left\{\mathrm{M}_{m}\right\}$ and two density operators $\rho$ and $\omega$, we rearrange POVM elements with respect to the decreasing order of numbers $\sqrt{p_{m} q_{m}} \downarrow$. Let us define new positive operator

$$
\mathrm{T}_{M}=\sum_{m>k} \mathrm{M}_{m}^{\downarrow} .
$$

Due to the completeness relation, one obeys $\mathrm{T}_{M} \leq \mathbf{1}$. Each of $(d-k)$ terms in Eq. (24) has trace exceeding one, whence $\operatorname{tr}\left(\mathrm{T}_{M}\right) \geq d-k$. Due to Eqs. (21) and (23), we can write

$$
F_{k}(\rho, \omega) \leq \operatorname{tr}\left(\mathrm{T}_{M} \sqrt{\omega} \vee \sqrt{\rho}\right)=\sum_{m>k} \operatorname{tr}\left(\sqrt{\rho} \mathrm{M}_{m}^{\downarrow} \sqrt{\omega} \mathrm{V}\right)
$$

Let us define operators $\mathrm{A}_{m}=\left(\mathrm{M}_{m}^{\downarrow}\right)^{1 / 2} \rho^{1 / 2}$ and $\mathrm{B}_{m}=\left(\mathrm{M}_{m}^{\downarrow}\right)^{1 / 2} \omega^{1 / 2} \mathrm{~V}$. In Eq. (25) each term of the sum is the Hilbert-Schmidt inner product $\left\langle A_{m}, B_{m}\right\rangle_{\text {hs }}$ satisfying

$$
\left|\left\langle\mathrm{A}_{m}, \mathrm{~B}_{m}\right\rangle_{\mathrm{hs}}\right| \leq\left\langle\mathrm{A}_{m}, \mathrm{~A}_{m}\right\rangle_{\mathrm{hs}}^{1 / 2}\left\langle\mathrm{~B}_{m}, \mathrm{~B}_{m}\right\rangle_{\mathrm{hs}}^{1 / 2} .
$$

Using the cyclic property of the trace, we have

$$
\begin{aligned}
& \left\langle\mathrm{A}_{m}, \mathrm{~A}_{m}\right\rangle_{\mathrm{hs}}=\operatorname{tr}\left(\mathrm{M}_{m}^{\downarrow} \rho\right), \\
& \left\langle\mathrm{B}_{m}, \mathrm{~B}_{m}\right\rangle_{\mathrm{hs}}=\operatorname{tr}\left(\mathrm{M}_{m}^{\downarrow} \omega\right) .
\end{aligned}
$$

Together with Eq. (26), the last equalities imply that

$$
\sum_{m>k}\left|\operatorname{tr}\left(\sqrt{\rho} \mathrm{M}_{m}^{\downarrow} \sqrt{\omega} \mathrm{V}\right)\right| \leq \sum_{m>k} \sqrt{p_{m} q_{m}} \downarrow
$$

Inequalities (25) and (29) provide Eq. (22) for every POVM whose elements fulfill the precondition $\operatorname{tr}\left(\mathrm{M}_{m}\right) \geq 1$.

For the 0-fidelity, the lower bound $\mathcal{F}_{0}\left(p_{m}, q_{m}\right)$ can be reached by some POVM's [4, 19]. For invertible $\rho$, the optimal POVM is formed by one-rank projectors $\mathbf{P}_{m}=|m\rangle\langle m|$, where $|m\rangle$ is eigenstate of positive operator $\mathbf{R}=$ $\rho^{-1 / 2}|\sqrt{\rho} \sqrt{\omega}| \rho^{-1 / 2}$. Here we have a proportionality

$$
\lambda_{m}(\mathrm{R}) \mathrm{P}_{m} \sqrt{\rho}=\mathrm{P}_{m} \sqrt{\omega} \mathrm{V} .
$$

Therefore, the Schwarz inequality (26) is fulfilled with equality for all $m$. However, this is not sufficient for partial fidelity of order $k>0$. To saturate inequality (22), we must also provide the equality in Eq. (25). But the operator

$$
\mathrm{T}_{P}=\sum_{m>k} \mathrm{P}_{m}^{\downarrow}
$$


is not the projector $\Pi_{\min }$ with necessity. When $\mathrm{T}_{P}=\Pi_{\min }$ for all $k$, the operators $\mathrm{R}$ and $|\sqrt{\rho} \sqrt{\omega}|$ are simultaneously diagonalizable. Thus, the operators $\mathrm{R}$ and $|\sqrt{\rho} \sqrt{\omega}|^{2}=\sqrt{\rho} \omega \sqrt{\rho}$ are commuting, whence

$$
\rho \omega|\sqrt{\rho} \sqrt{\omega}|=|\sqrt{\rho} \sqrt{\omega}| \rho \omega .
$$

So we have arrived at a conclusion. If the inequality (22) can be saturated for all $k=0, \ldots, d-1$, then the operators $\rho \omega$ and $|\sqrt{\rho} \sqrt{\omega}|$ are commuting.

It should be pointed out that the precondition $\operatorname{tr}\left(\mathrm{M}_{m}\right) \geq 1$ of Theorem 4 is necessary. Indeed, for POVM with several elements of the form $\mathrm{N}_{m}=\epsilon|m\rangle\langle m|$ proper fidelities $\mathcal{F}_{k}\left(p_{m}, q_{m}\right)$ can be made arbitrarily small independently of $F_{k}(\rho, \omega)$. But such POVM's can unlikely be interesting in the practice. For many applications of quantum information we primarily deal with projective measurements 4. The statement of Theorem 4 holds for all the projective measurements. From the operational point of view, the attainability of the lower bound in Eq. (22) is hardly essential. In quantum information processing, we usually have only partial knowledge about quantum states. So the measurement optimizing Eq. (19) cannot be found a priori. In this situation the right-hand sides of Eqs. (19) and (22) provide measurable estimates from above on the quantum fidelities.

\section{Sub-multiplicativity and relation with trace distances}

It is usual in the study of quantum information that one deals with composite systems 4, 17. Here the multiplicativity of fidelity makes this measure very convenient to use. That is, if $\rho$ and $\omega$ are density operators on $\mathcal{H}_{A}, \Theta$ and $\Omega$ are density operators on $\mathcal{H}_{E}$, then

$$
F_{0}(\rho \otimes \Theta, \omega \otimes \Omega)=F_{0}(\rho, \omega) F_{0}(\Theta, \Omega) .
$$

The authors of Ref. 13 introduced two quantity, namely sub-fidelity and superfidelity. These measure are easier to experimental measurement than the fidelity. The sub-fidelity and super-fidelity provide the lower and upper bounds on the fidelity respectively. In terms of super-fidelity the strong lower bound on the trace distance has been established [26]. The sub-fidelity is sub-multiplicative, the super-fidelity is super-multiplicative [13. We shall now find analogous property for the partial fidelities.

There is simple property of singular values with respect to the operation of tensor product [17. Namely, the singular values of tensor product of two operators are the products of all pairs consisting of a singular value of first and a singular value of second operator. A justification is the following. Recall that for arbitrary four operators of proper dimensionality there holds [17.

$$
(\mathrm{X} \otimes \Theta)(\mathrm{Y} \otimes \Omega)=(\mathrm{XY}) \otimes(\Theta \Omega) .
$$

Using this property and $\sqrt{\mathrm{X} \otimes \Theta}=\sqrt{\mathrm{X}} \otimes \sqrt{\Theta}$, we at once obtain

$$
|X \otimes \Theta|=\sqrt{(X \otimes \Theta)^{\dagger}(X \otimes \Theta)}=\sqrt{\left(X^{\dagger} X\right) \otimes\left(\Theta^{\dagger} \Theta\right)}=|X| \otimes|\Theta| .
$$


In the same manner, we also have

$$
\sqrt{\rho \otimes \Theta} \sqrt{\omega \otimes \Omega}=\sqrt{\rho} \sqrt{\omega} \otimes \sqrt{\Theta} \sqrt{\Omega} .
$$

Let $|x\rangle$ be eigenvector of $|X|$, and let $|\theta\rangle$ be eigenvector of $|\Theta|$. By Eq. (35), the product $|x\rangle \otimes|\theta\rangle$ is eigenvector of $|\mathbf{X} \otimes \Theta|$. So we get the following. If $d$ numbers $s_{j}(\mathrm{X})$ are singular values of operator $\mathrm{X}, N$ numbers $s_{i}(\Theta)$ are singular values of operator $\Theta$, then $d N$ products

$$
s_{j i}(\mathrm{X} \otimes \Theta)=s_{j}(\mathrm{X}) s_{i}(\Theta)
$$

give all the singular values of operator $\mathrm{X} \otimes \Theta$ in a priori unknown order. In accordance with Definition 1, we have

$$
F_{(d-k)}(\rho, \omega) F_{(N-L)}(\Theta, \Omega)=\sum_{j=d-k+1}^{d} s_{j}^{\downarrow}(\sqrt{\rho} \sqrt{\omega}) \sum_{i=N-L+1}^{N} s_{i}^{\downarrow}(\sqrt{\Theta} \sqrt{\Omega}) .
$$

Due to Eqs. (35), (36) and (37), the right-hand side of Eq. (38) keeps a sum of $k L$ singular values of operator $\sqrt{\rho \otimes \Theta} \sqrt{\omega \otimes \Omega}$. The partial fidelity of order $(d N-k L)$ between $\rho \otimes \Theta$ and $\omega \otimes \Omega$ does not exceed this sum. So, there holds

$$
F_{(d N-k L)}(\rho \otimes \Theta, \omega \otimes \Omega) \leq F_{(d-k)}(\rho, \omega) F_{(N-L)}(\Theta, \Omega) .
$$

Therefore, the partial fidelities are sub-multiplicative in the sense of Eq. (39). When $k=d$ and $L=N$, the right-hand side of Eq. (38) summarized all the singular values of operator $\sqrt{\rho \otimes \Theta} \sqrt{\omega \otimes \Omega}$. So, the inequality (39) is replaced by Eq. (33). Thus, the multiplicative property of partial fidelities is more complicated in character. In general, this is not unexpected.

On a level with properties of some measure itself, the relationships between different measures of distinguishability are very interesting. It is well-known that the fidelity and the trace distance are related by the inequalities 28.

$$
1-F(\rho, \omega) \leq D(\rho, \omega) \leq \sqrt{1-F(\rho, \omega)^{2}} .
$$

Here the trace distance between density operators $\rho$ and $\omega$ is defined by

$$
D(\rho, \omega):=\frac{1}{2} \operatorname{tr}|\rho-\omega| .
$$

There is an alternative definition via extremal properties of quantum operations [29]. Therefore, these measures may be considered to be equivalent for many applications 4, 17. A relative analog of the above relationship can be obtained in terms of partitioned trace distances. In Ref. [27, the present author imported this family of new distances between mixed quantum states. For $k=1, \ldots, d$, the Ky Fan $k$-norm of operator $\mathrm{X}$ is defined by [18]

$$
\|\mathrm{X}\|_{(k)}:=\sum_{j=1}^{k} s_{j}(\mathrm{X}),
$$

where singular values are assumed to be indexed in decreasing order. The $k$-th partitioned trace distance between density operator $\rho$ and $\omega$ is introduced as

$$
D_{k}(\rho, \omega):=\frac{1}{2}\|\rho-\omega\|_{(k)} .
$$


The partitioned distances succeed many properties of the standard trace distance, namely the unitary invariance, the strong convexity and the bounds. In Ref. [27, the present author also defined the $k$-th classical distance between two probability distributions $\left\{p_{i}\right\}$ and $\left\{q_{i}\right\}$ as

$$
\mathcal{D}_{k}\left(p_{i}, q_{i}\right):=\frac{1}{2} \sum_{i=1}^{k}\left|p_{i}-q_{i}\right|^{\downarrow}
$$

where the arrows down indicate that the absolute values are put in the decreasing order. Using the Ky Fan maximum principle, we can prove that [27]

$$
D_{k}(\rho, \omega)=\max \left\{\mathcal{D}_{k}\left(p_{m}, q_{m}\right): \operatorname{tr}\left(\mathrm{M}_{m}\right) \leq 1\right\},
$$

where the maximum is taken over all POVMs $\left\{M_{m}\right\}$ whose elements satisfy $\operatorname{tr}\left(\mathrm{M}_{m}\right) \leq 1$. The maximum in Eq. (45) is reached by one-rank PVM $\left\{Q_{m}\right\}$ associated with the Jordan decomposition of difference $(\rho-\omega)$ 27]. We rearrange elements of this optimal PVM with respect to the decreasing orders of numbers $\left|p_{m}-q_{m}\right|^{\downarrow}$. Let us define projector $\Pi^{\prime}$ of rank $(d-k)$ by

$$
\Pi^{\prime}:=\sum_{m>k} \mathrm{Q}_{m}^{\downarrow}
$$

Putting $X=\Pi^{\prime}$ and $Y=\Pi^{\prime}$, we satisfy Eqs. (4) and (5). Due to Eq. (6), we then have

$$
\begin{aligned}
2 F_{k}(\rho, \omega) & \leq \operatorname{tr}\left(\Pi^{\prime} \rho\right)+\operatorname{tr}\left(\Pi^{\prime} \omega\right) \\
& =1-\sum_{m=1}^{k} p_{m}+1-\sum_{m=1}^{k} q_{m} \\
& \leq 2-\sum_{m=1}^{k}\left|p_{m}-q_{m}\right|^{\downarrow} .
\end{aligned}
$$

By choice of optimal PVM, the right-hand side of Eq. (47) is equal to quantity $\left(2-2 D_{k}(\rho, \omega)\right)$. Hence we obtain

$$
F_{k}(\rho, \omega)+D_{k}(\rho, \omega) \leq 1 .
$$

This inequality should not be confused with inequalities (40) which contain $F_{0}(\rho, \omega)$ and $D_{d}(\rho, \omega)$. Using Eq. (15), we easily derive that

$$
\frac{k}{r} \mathcal{D}_{r}\left(p_{i}, q_{i}\right) \leq \mathcal{D}_{k}\left(p_{i}, q_{i}\right), \quad \frac{k}{d} D_{d}(\rho, \omega) \leq D_{k}(\rho, \omega) .
$$

By Eq. (17), the $k$-th partial fidelity is bounded from above. By Eq. (49), the $k$ th partitioned trace distance is bounded from below. So the inequality (48) is not trivial, although it differs from inequalities (40) in character. Thus, if the $k$-th partial fidelity between two states is close to one then the $k$-th partitioned trace distance is close to zero. In the mentioned sense, these measures of closeness for quantum states can be regarded as equivalent.

\section{Monotonicity under unistochastic quantum op- erations}

One of the basic properties of the fidelity is that fidelity cannot decrease under trace-preserving quantum operation 25]. This property is usually referred to 
as the monotonicity under quantum operations 4. In this section we show that the partial fidelities possess the same monotonicity but with respect to unistochastic operations. From the physical point of view, the most general operation on the principal system $A$ is to allow $A$ to interact unitarily with $N$-dimensional environment $E$ in a standard (normalized) state. The final state of $A$ is then obtained by the operation of partial trace. Let $\Theta$ denote the initial standard state of environment. A linear map $\mathcal{E}$ is defined by

$$
\mathcal{E}(\rho):=\operatorname{tr}_{E}\left(\mathrm{U}(\rho \otimes \Theta) \mathrm{U}^{\dagger}\right),
$$

where unitary operator $U$ acts on the space $\mathcal{H}_{A} \otimes \mathcal{H}_{E}$ and the trace is taken over space $\mathcal{H}_{E}$ of environment. This is environmental representation of the map [7. It is commonly to check that the above map is linear and completely positive. Due to the properties of the trace and $\operatorname{tr}_{E}(\Theta)=1$, one also holds

$$
\operatorname{tr}_{A}(\mathcal{E}(\rho))=\operatorname{tr}_{A}(\rho) \operatorname{tr}_{E}(\Theta)=\operatorname{tr}_{A}(\rho) .
$$

So the map $\mathcal{E}$ is trace-preserving. For all the normalized inputs $\rho$, we have $\operatorname{tr}_{A}(\mathcal{E}(\rho))=1$. This gives the probability that the described physical process occurs. Thus, the considered process is deterministic. Trace-preserving completely positive maps are known under various names [7] deterministic quantum operations, quantum channels, stochastic maps. The important case of Eq. (50) is given, when the initial state of the environment is maximally mixed, $\Theta=\mathbf{1}_{E} / N$. Such quantum channels are called unistochastic [7. Their physical reason is natural. With no knowledge about the environment, one assumes that it is initially in the maximally mixed state. Unistochastic quantum operation is analog of classical transformations given by unistochastic matrices [7. It is clear that unistochastic channels leave the maximally mixed state $\mathbf{1}_{A} / d$ invariant. So they are unital maps. In the one-qubit case, both the depolarizing and phase damping channels are unistochastic.

Like the fidelity [25], the monotonicity of partial fidelities is based on their behaviour under the operation of partial trace. Let $\rho_{A}$ and $\omega_{A}$ be density operators obtained as traces

$$
\begin{aligned}
\rho_{A} & =\operatorname{tr}_{E}(\widetilde{\rho}), \\
\omega_{A} & =\operatorname{tr}_{E}(\widetilde{\omega}),
\end{aligned}
$$

from density operators $\widetilde{\rho}$ and $\widetilde{\omega}$ on the space $\mathcal{H}_{A} \otimes \mathcal{H}_{E}$. To find the necessary property, we consider the quantity

$$
G_{k}\left(\rho_{A}, \omega_{A} \mid \mathrm{X}, \mathrm{Y}\right)=\operatorname{tr}_{A}\left(\rho_{A} \mathrm{X}\right)+\operatorname{tr}_{A}\left(\omega_{A} \mathrm{Y}\right)
$$

where positive operators $X$ and $Y$ obey Eqs. (4) and (5), $\operatorname{rank}(X)=\operatorname{rank}(Y)=$ $d-k$. We now put the operators

$$
\begin{aligned}
& \widetilde{X}:=\mathrm{X} \otimes \mathbf{1}_{E}, \\
& \widetilde{\mathrm{Y}}:=\mathrm{Y} \otimes \mathbf{1}_{E},
\end{aligned}
$$

$\underset{\widetilde{Y}}{\text { with }} \widetilde{Y}$ the identity $\mathbf{1}_{E}$ on the environment space. They satisfy $\widetilde{X} \widetilde{Y} \widetilde{X}=\widetilde{X}$ and $\widetilde{Y} \widetilde{X} \widetilde{Y}=\widetilde{Y}$. Due to the properties of partial trace [4, 17], we have

$$
\begin{aligned}
& \operatorname{tr}\left(\widetilde{\rho}\left(\mathrm{X} \otimes \mathbf{1}_{E}\right)\right)=\operatorname{tr}_{A}\left(\operatorname{tr}_{E}(\widetilde{\rho}) \mathrm{X}\right), \\
& \operatorname{tr}\left(\widetilde{\omega}\left(\mathrm{Y} \otimes \mathbf{1}_{E}\right)\right)=\operatorname{tr}_{A}\left(\operatorname{tr}_{E}(\widetilde{\omega}) \mathrm{Y}\right) .
\end{aligned}
$$


Therefore, the quantity $G_{k}\left(\rho_{A}, \omega_{A} \mid \mathrm{X}, \mathrm{Y}\right)$ is equal to the quantity

$$
G_{(k N)}(\widetilde{\rho}, \widetilde{\omega} \mid \widetilde{X}, \widetilde{Y})=\operatorname{tr}(\widetilde{\rho} \widetilde{\mathrm{X}})+\operatorname{tr}(\widetilde{\omega} \widetilde{\mathrm{Y}}) .
$$

Hence we obtain the equality

$$
\inf \left\{G_{k}\left(\rho_{A}, \omega_{A} \mid \mathrm{X}, \mathrm{Y}\right)\right\}=\inf \left\{G_{(k N)}(\widetilde{\rho}, \widetilde{\omega} \mid \widetilde{\mathrm{X}}, \widetilde{\mathrm{Y}}): \widetilde{\mathrm{X}}=\mathrm{X} \otimes \mathbf{1}_{E}, \widetilde{\mathrm{Y}}=\mathrm{Y} \otimes \mathbf{1}_{E}\right\}
$$

We also note that $\operatorname{rank}(\widetilde{\mathrm{X}})=\operatorname{rank}(\widetilde{\mathrm{Y}})=(d-k) N$. So we can apply the Uhlmann result (6) to both the sides of Eq. (60). The left-hand side of Eq. (60) is doubled $k$-fidelity $F_{k}\left(\rho_{A}, \omega_{A}\right)$. The right-hand side of Eq. (60) is conditional infimum which is larger than or equal to $2 F_{(k N)}(\widetilde{\rho}, \widetilde{\omega})$. That is, we have proved

$$
F_{k}\left(\operatorname{tr}_{E}(\widetilde{\rho}), \operatorname{tr}_{E}(\widetilde{\omega})\right) \geq F_{(k N)}(\widetilde{\rho}, \widetilde{\omega}) .
$$

For $k=0$ we have the well-known result that the fidelity cannot decrease under operation of partial trace [25]. This provides the intuitive reason that objects become less distinguishable when only partial information is available [4. In general, the partial fidelities enjoy less property. But we are able to establish the following result.

Theorem 5. If the map $\mathcal{E}$ is unistochastic then for all $k=0,1, \ldots, d-1$ and any two inputs $\rho$ and $\omega$ there holds

$$
F_{k}(\mathcal{E}(\rho), \mathcal{E}(\omega)) \geq F_{k}(\rho, \omega) .
$$

Proof. Each unistochastic operation can be written in the form (50) with $\Theta=\mathbf{1}_{E} / N$. Using the multiplicative properties, we see that singular values

$$
s_{j i}(\sqrt{\rho \otimes \Theta} \sqrt{\omega \otimes \Theta})=s_{j}(\sqrt{\rho} \sqrt{\omega}) s_{i}(\Theta)=\frac{1}{N} s_{j}(\sqrt{\rho} \sqrt{\omega}) .
$$

Hence the sum of $(d-k) N$ smallest numbers $s_{j i}$ is equal to the sum of $(d-k)$ smallest numbers $s_{j}(\sqrt{\rho} \sqrt{\omega})$, that is

$$
F_{(k N)}(\rho \otimes \Theta, \omega \otimes \Theta)=F_{k}(\rho, \omega) .
$$

Due to the unitary invariance, we have

$$
F_{(k N)}(\rho \otimes \Theta, \omega \otimes \Theta)=F_{(k N)}\left(\mathrm{U}(\rho \otimes \Theta) \mathrm{U}^{\dagger}, \mathrm{U}(\omega \otimes \Theta) \mathrm{U}^{\dagger}\right) .
$$

By partial trace operation and Eq. (61), we obtain Eq. (62).

Note that the choice $\Theta=\mathbf{1}_{E} / N$ is necessary for validity of Eq. (64) for all inputs. Indeed, there is no reasons to think that partial fidelities enjoy the whole of properties of the fidelity. We now show that the monotonicity of partial fidelities is violated under the amplitude damping with $\gamma \in(0 ; 1)$. In the Bloch representation, the effect of amplitude damping is expressed as [4]

$$
\left(u_{x}, u_{y}, u_{z}\right) \longmapsto\left(u_{x} \sqrt{1-\gamma}, u_{y} \sqrt{1-\gamma}, \gamma+u_{z}(1-\gamma)\right) .
$$

Here $\vec{u}$ denotes the Bloch vector of input density matrix. Choose two inputs $\rho=(1 / 2)\left\{\mathbf{1}+v \sigma_{z}\right\}$ and $\omega=(1 / 2)\left\{\mathbf{1}+w \sigma_{z}\right\}$, where $\sigma_{z}$ is the Pauli matrix and $v, w \in(0 ; 1)$. By calculations, the smallest eigenvalue of $|\sqrt{\rho} \sqrt{\omega}|$ is equal to $\sqrt{(1-v)(1-w)} / 2=F_{1}(\rho, \omega)$. As a result of amplitude damping, the outputs 
$\rho^{\prime}=(1 / 2)\left\{\mathbf{1}+v^{\prime} \sigma_{z}\right\}$ and $\omega^{\prime}=(1 / 2)\left\{\mathbf{1}+w^{\prime} \sigma_{z}\right\}$ have the Bloch vectors $\left(0,0, v^{\prime}\right)$ and $\left(0,0, w^{\prime}\right)$ respectively, where

$$
\begin{gathered}
v^{\prime}=\gamma+v(1-\gamma), \\
w^{\prime}=\gamma+w(1-\gamma),
\end{gathered}
$$

in line with Eq. (66). By analogy, we get $F_{1}\left(\rho^{\prime}, \omega^{\prime}\right)=\sqrt{\left(1-v^{\prime}\right)\left(1-w^{\prime}\right)} / 2$. It is easy to check that (except $v=1$ and $w=1$ )

$$
\left(1-v^{\prime}\right)\left(1-w^{\prime}\right)<(1-v)(1-w)
$$

and, therefore, $F_{1}\left(\rho^{\prime}, \omega^{\prime}\right)<F_{1}(\rho, \omega)$. The violation of Eq. (62) is natural because the amplitude damping channel is not unistochastic. Meanwhile, partial fidelity $F_{1}$ can be increased under action of amplitude damping. The maximally mixed state $\rho_{*}=\mathbf{1} / 2$ is transformed into $\rho^{\prime \prime}=(1 / 2)\left\{\mathbf{1}+\gamma \sigma_{z}\right\}$. We also take state $\omega_{*}=(1 / 2)\left\{\mathbf{1}-\alpha \sigma_{z}\right\}$ with $\alpha=\gamma /(1-\gamma)$ so that it is mapped into the maximally mixed state, i.e. $\omega^{\prime \prime}=1 / 2$. To provide $\alpha \leq 1$, we assume that $\gamma \leq 1 / 2$. By simple calculations, we get $F_{1}\left(\rho_{*}, \omega_{*}\right)=\sqrt{1-\alpha} / 2$ and $F_{1}\left(\rho^{\prime \prime}, \omega^{\prime \prime}\right)=\sqrt{1-\gamma} / 2$. It is clear that $\gamma<\alpha$ and $F_{1}\left(\rho^{\prime \prime}, \omega^{\prime \prime}\right)>F_{1}\left(\rho_{*}, \omega_{*}\right)$ as claimed. Thus, the monotonicity of partial fidelities is violated under the action of some stochastic maps. As it is shown in Ref. 27, the partitioned trace distances cannot increase under quantum operations of certain kind including bistochastic maps. Similarly, the partial fidelities are monotone with respect to subclass of trace-preserving quantum operations.

\section{Conclusion}

We have analyzed some important properties of Uhlmann's partial fidelities. The equality of the standard fidelity for two pairs of density operators does not imply their unitary equivalence. It is for this reason that the partial fidelities were introduced by Uhlmann. We have obtained simple bounds on $k$-th partial fidelity. Quantum-classical relations are considered in terms of quantum measurement. Like usual fidelity, this gives a statistical interpretation for partial fidelity. The relationship with partitioned trace distances is obtained. Our reasons are significantly based on the Ky Fan maximum principle and its consequences. In a certain sense, the partial fidelities are sub-multiplicative. It is shown that any partial fidelity cannot decrease under unistochastic quantum operation. That is, it enjoys monotonicity. In general, however, the partial fidelities are not monotone. The derived properties allow to lighten work with the partial fidelities.

\section{References}

[1] A. Uhlmann (1976), The transition probability in the state space of a ${ }^{*}$ algebra, Rep. Math. Phys. 9, pp. 273-279.

[2] P. M. Alberti and A. Uhlmann (1983), Stochastic linear maps and transition probability, Lett. Math. Phys. 7, pp. 107-112.

[3] R. Jozsa (1994), Fidelity for mixed quantum states, J. Mod. Opt. 41, pp. 2315-2323. 
[4] M. A. Nielsen and I. L. Chuang (2000), Quantum computation and quantum information, Cambridge University Press (Cambridge).

[5] A. Gilchrist, N. K. Langford and M. A. Nielsen (2005), Distance measures to compare real and ideal quantum processes, Phys. Rev. A 71, 062310.

[6] S. L. Braunstein and C. M. Caves (1995), Geometry of quantum states, Proceedings of the Conference on Quantum Communication and Measurement, edited by R. Hudson, V. P. Belavkin, O. Hirota, New York (Plenum Press), pp. 21-30.

[7] I. Bengtsson and K. Życzkowski (2006), Geometry of quantum states: an introduction to quantum entanglement, Cambridge University Press (Cambridge).

[8] V. Giovannetti and A. S. Holevo (2008), Quantum Shared Broadcasting, Quantum Information Processing 7, pp. 55-69.

[9] M. R. Dowling and M. A. Nielsen (2008), The geometry of quantum computation, Quantum Information \& Computation 8, pp. 861-899.

[10] C. A. Fuchs (1996), Distinguishability and accessible information in quantum theory, quant-ph/9601020.

[11] A. Uhlmann (2000), On "partial" fidelities, Rep. Math. Phys. 45, pp. 407418.

[12] P. E. M. F. Mendonça, R. J. Napolitano, M. A. Marchiolli, C. J. Foster and Y.-C. Liang (2008), Alternative fidelity measure between quantum states, Phys. Rev. A 78, 052330.

[13] J. A. Miszczak, Z. Puchała, P. Horodecki, A. Uhlmann and K. Życzkowski (2009), Sub- and super-fidelity as bounds for quantum fidelity, Quantum Information \& Computation 9, pp. 0103-0130.

[14] A. E. Rastegin (2003), Upper bound on the global fidelity for mixed-state cloning, Phys. Rev. A 68, 012305.

[15] K. Życzkowski and W. Słomczyński (1998), The Monge distance between quantum states, J. Phys. A: Math. Gen. 31, pp. 9095-9104.

[16] A. E. Rastegin (2006), Sine distance for quantum states, quant-ph/0602112.

[17] J. Watrous (2008), CS 798: Theory of quantum information, University of Waterloo, http://www.cs.uwaterloo.ca/ watrous/quant-info/lecture-notes/all-lectures.pdf

[18] R. Bhatia (1997), Matrix Analysis, Springer (New York).

[19] C. A. Fuchs and C. M. Caves (1995), Mathematical techniques for quantum communication theory, Open Systems \& Information Dynamics 3, pp. 345356.

[20] A. E. Rastegin (2003), Global-fidelity limits of state-dependent cloning of mixed states, Phys. Rev. A 68, 032303. 
[21] P. M. Alberti and A. Uhlmann (2000), On Bures distance and ${ }^{*}-$ algebraic transition probability between inner derived positive linear forms over $W^{*}$ algebras, Acta Applicandae Mathematicae 60, pp. 1-37.

[22] A. Peres (1993), Quantum theory: concepts and methods, Kluwer (Dordrecht).

[23] K. Fan (1949), On a theorem of Weyl concerning eigenvalues of linear transformations. I, Proc. Nat. Acad. Sci. U.S.A. 35, pp. 652-655.

[24] M. A. Nielsen and G. Vidal (2001), Majorization and the interconversion of bipartite states, Quantum Information \& Computation 1, pp. 76-93.

[25] H. Barnum, C. M. Caves, C. A. Fuchs, R. Jozsa and B. Schumacher (1996), Noncommuting mixed states cannot be broadcast, Phys. Rev. Lett. 76, pp. $2818-2821$.

[26] Z. Puchała and J. A. Miszczak (2009), Bound on trace distance based on super-fidelity, Phys. Rev. A 76, 024302.

[27] A. E. Rastegin (2009), Partitioned trace distances, arXiv:0903.4543 [quant$\mathrm{ph}$.

[28] C. A. Fuchs and J. van de Graaf (1999), Cryptographic distinguishability measures for quantum mechanical states, IEEE Trans. Inf. Theory 45, pp. 1216-1227.

[29] A. E. Rastegin (2007), Trace distance from the viewpoint of quantum operation techniques, J. Phys. A: Math. Theor. 40, 9533-9549. 